\title{
Graphene-containing flowable electrodes for capacitive energy storage
}

\author{
M. Boota ${ }^{\mathrm{a}}$, K. B. Hatzell ${ }^{\mathrm{a}}$, M. Alhabeb ${ }^{\mathrm{a}}$, E. C. Kumbur ${ }^{\mathrm{b}}$, and Y. Gogotsi ${ }^{\mathrm{a}^{*}}$ \\ ${ }^{a}$ A. J. Drexel Nanomaterials Institute and Department of Materials Science and Engineering, Drexel \\ University, Philadelphia, PA 19104, USA \\ ${ }^{\mathrm{b}}$ Electrochemical Energy Systems Laboratory, Department of Mechanical Engineering and Mechanics, \\ Drexel University, Philadelphia, PA 19104, USA
}

\begin{abstract}
High conductivity and extended particle contacts are required for rapid charge percolation in flowable electrodes. In this study, carbon spheres (CS) were wrapped by highly conductive reduced graphene oxide sheets (rGO) to address these issues. Various compositions of the conductive, 3D interconnected hybrid materials (rGO@CS) were synthesized by a hydrothermal method. Synergistic effects of both materials were utilized where CS served to minimize the sheet stacking for better flowability of the suspensions, and wrapped rGO sheets enabled higher conductivity for fast charge transport throughout the suspension network. When tested as flowable electrodes, the composition with a 1:2 ratio of GO to CS exhibited the highest capacitance of $200 \mathrm{~F} / \mathrm{g}$ and an improved rate performance. The improved performance is attributed to the fast charge transport in the suspension network due to higher conductivity and enhanced connectivity of the active material particles. Optimized electrodes were also examined in a flow mode which yielded a capacitance of $45 \mathrm{~F} / \mathrm{g}$.
\end{abstract}

Keywords: carbon; graphene; flow capacitor; flowable electrode; supercapacitor

\footnotetext{
*Corresponding author. Tel: 215895 6446. E-mail: gogotsi@drexel.edu (Yury Gogotsi)
} 


\section{Introduction}

The growing interest to renewable energy generation from intermittent sources, such as solar, wind, and hydro, requires the development of environmentally benign and inexpensive electrical energy storage systems for the power grid. Recently, a new family of flowable electrochemical energy storage systems based on suspension electrodes has emerged as an alternative technology for large-scale energy storage. In contrast to static energy storage devices (e.g., supercapacitors and lithium ion batteries) confined to small cells, electrochemical systems based on flowable electrodes benefit from the versatility of a flow-architecture. Specifically, this feature enables the decoupling of power and energy capacity for scalable systems with design flexibility. [1-3]

A flowable electrode, also called semi-solid [4], suspension [5] or slurry electrode [6], is a composed of an active (charge storing) material, a conductive additive, and an electrolyte. The conducting and connecting framework of active material enables charge and ion percolation throughout the thickness of the electrode. Depending on the active material used, flowable electrodes either store charge electrostatically in an electric double layer (capacitive) [2] or via faradic (redox) mechanisms. The widespread application of faradic flow-assisted technologies (e.g., redox flow batteries, [7] semi-solid lithium ion batteries [4] etc.) is hindered due to inherent challenges such as slow kinetics, use of expensive materials (e.g., lithium, vanadium, etc.) and operation in non-aqueous environment. [4,8,9] In contrast, capacitive flowable electrodes are gaining interest as they utilize inexpensive and abundant materials, mainly activated carbons in aqueous electrolytes. $[2,10,11]$ The electrochemical flow capacitor (EFC) $[2,11]$ is an example of a new technology (based on capacitive suspension electrodes) being examined for grid scale energy storage. Since the EFC stores charges electrostatically (similar to 
supercapacitors) it offers fast charging/discharging, longer cycle life and a scalable architecture. During operation, flowable electrodes are pumped in batches into the flow cell, where they are charged in static mode. After completely charged, the slurry is then pumped out of the cell and stored in different tanks. When energy is required, the slurry can be pumped back into the cell and discharged. Further details of the EFC operation can be found in our previous publications. $[2,12,13]$

Recently, different types of the carbon materials (e.g., spherical and anisotropic activated carbon, carbide derived carbon, etc.), metal oxides and redox molecules have been used as electroactive materials. [5,13-18] In our previous studies, one key issue observed was the deterioration of the slurry performance at scan rates of $10-20 \mathrm{mV} / \mathrm{s}$, due to the poor conductivity and particle connectivity within the slurries. In line with this observation, one key finding was that the design of the high performing flowable (suspension-type) electrodes requires a balanced approach. Specifically, high conductivity, enhanced rheology (low-viscosity), and excellent electrochemical performance must be achieved.

Reduced graphene oxide (rGO) sheets display high conductivity, moderate capacitances, higher mechanical strength and have been widely studied by the energy storage and polymer composite community. [10,19-22] Their sheet morphology and high conductivity are of interest for flowable electrode systems that require high connectivity between active materials. However, it is difficult to make flowable electrodes having balanced rheological and electrochemical characteristics based on the 2D rGO sheets due to various reasons, such as sheet stacking in the electrolytes, high viscosity (poor flowability of the slurry), and strong aggregation in aqueous electrolytes. [23] One way to tackle these challenges is to combine rGO sheets with 100-250 $\mu \mathrm{m}$ size spherical carbons. Our hypothesis was that GO sheets can wrap around the 
Table 1: Comparison of the rGO@CS with the best previously reported flowable electrodes under intermittent flow conditions.

\begin{tabular}{ccccc}
\hline $\begin{array}{c}\text { Type of carbon } \\
\text { material }\end{array}$ & Charge time & $\begin{array}{c}\text { Gravimetric } \\
\text { Capacitance } \\
(\mathbf{F} / \mathbf{g})\end{array}$ & $\begin{array}{c}\text { Volumetric } \\
\text { Capacitance } \\
(\mathbf{F} / \mathbf{m L})\end{array}$ & Reference \\
\hline YP-50 & $20 \mathrm{~min}$ & 17 & 3.3 & {$[6][14]$} \\
CS-1000 & $20 \mathrm{~min}$ & 30 & 6.9 & {$[14]$} \\
rGO@CS & $20 \mathrm{~min}$ & 45 & 9.1 & Current Work \\
\hline
\end{tabular}


micron-scaled CS due to van der Waals interactions, and thus would allow us to achieve a good dispersion of rGO sheets (for high conductivity), while still maintaining a high specific surface area (for electrical double layer charge storage). Moreover, it is anticipated that the rGO sheets will enhance the conductivity of the slurries and improve connectivity between particles for facile charge percolation.

In this study, we present a strategy for effective utilization of rGO sheets in suspension based flowable electrodes having balanced mass loadings, conductivity, connectivity and rheology for electrochemical flow capacitors. A comprehensive analysis of the physical and electrochemical characteristics of hybrid flowable electrodes with various compositions was performed and advantages and limitations of using rGO sheets in slurries are discussed.

\section{Experimental Section}

\subsection{Synthesis of the hybrid electrodes (rGO@CS)}

All chemicals (analytical grade) were used as received without any further purification. Aqueous 1 M sulfuric acid (95-98 wt\%, Fischer) was used as the supporting electrolyte to make all the slurries for electrochemical testing. Carbon black (100\% compressed; Alfa Aesar, USA) was used as a conductive additive in each suspension electrode. Phenolic resin derived carbon spheres (125-250 $\mu \mathrm{m}$ in diameter) were purchased from MAST Carbon (United Kingdom). GO was synthesized by modified Hummers method. [24] Simultaneous GO reduction and wrapping around CS via self-assembly approach was performed through hydrothermal route. Varying amounts of GO to CS were added to synthesize different compositions of rGO@CS hybrids. In a typical synthesis of the 1:2 composition, a total of $50 \mathrm{ml}$ of $1 \mathrm{mg} / \mathrm{ml} \mathrm{GO}$ aqueous solution was added to $100 \mathrm{mg}$ CS. The mixture was bath sonicated for 1 hour, then transferred to $100 \mathrm{ml}$ capacity Teflon-lined hydrothermal container and heated to $150{ }^{\circ} \mathrm{C}$ for $6 \mathrm{~h}$. The autoclave was 
cooled down to room temperature resulting rGO@CS hybrid in the form of precipitates, which then filtered, washed several times with water and then oven dried overnight at $100{ }^{\circ} \mathrm{C}$ for $24 \mathrm{~h}$ before electrochemical testing.

\subsection{Preparation of the flowable electrodes}

To make flowable suspension electrodes, active material was mixed with carbon black (9:1) followed by the addition of $1 \mathrm{M} \mathrm{H}_{2} \mathrm{SO}_{4}$ to attain $23 \mathrm{wt} \%$ solid in the electrode, a predetermined weight percentage for the optimum electrochemical performance. [12] Few drops of water were added to assist the homogenous suspension formation, which later was stirred and heated at $\sim 60{ }^{\circ} \mathrm{C}$, until the evaporation of the extra added DI water.

\subsection{Structural characterizations}

Quadrosorb gas sorption instrument (Qunatachrome, USA) was used to determine specific surface area (SSA) and pore size distributions (PSD) by $\mathrm{N}_{2}$ gas sorption at $-196^{\circ} \mathrm{C}$. The average volume weighed pore size was determined using the cumulative pore volume by assuming slit-shaped pores and by using the quench-solid density functional theory (QSDFT) algorithm, similar to our previous publications. [5,12,14] A Zeiss Supra 50VP scanning electron microscope (Carl Zeiss AG, Germany) operating at $3 \mathrm{kV}$ was used to capture SEM images of the CS, rGO and their hybrids. Raman spectra of all the materials were recorded using Renishaw inVia spectrometer with a $632 \mathrm{~nm}$ laser as excitation source using $10 \%$ laser power in all the measurements. TGA was performed using TGA analyzer (TA instruments) under inert (Argon) atmosphere. Samples were first held to $150{ }^{\circ} \mathrm{C}$ for $1 \mathrm{~h}$ to evaporate adsorbed vapors or water molecules and then heated to $800{ }^{\circ} \mathrm{C}$ at $2.5{ }^{\circ} \mathrm{C} / \mathrm{min}$. FTIR spectra were recorded using Fouriertransform infrared spectrometer (Perkin-Elmer) with a resolution of $4 \mathrm{~cm}^{-1}$. 


\subsection{Electrochemical performance}

All electrochemical testing was performed on symmetric two-electrode cell with stainless steel current collectors. Channels of static cells were uniformly loaded with slurry separated by polyvinylidene fluoride (PVDF) membrane separator with an average pore size of $100 \mathrm{~nm}$ (Durapore®; Merck Millipore, Germany). Electrochemical performance was investigated using VMP3 potentiostat/galvanostat (BioLogic, France). Capacitance and rate performance was calculated from cyclic voltammograms. EIS which was performed in the frequency range of 200 $\mathrm{kHz}$ to $10 \mathrm{mHz}$ at a sine wave signal amplitude of $10 \mathrm{mV}$. Intermittent flow tests were performed on optimized composition of the flowable electrodes by pumping the uncharged slurry into the flow cell using syringe $(0.5 \mathrm{ml})$ which then potentiostatically charged at $1 \mathrm{~V}$ for $20 \mathrm{~min}$. The charged slurry was first pumped out of the cell, and then pumped back into the cell. Intermittent mode of operation is energetically favorable compared to the continuous mode, which is common in redox flow batteries. [25] The gravimetric and volumetric capacitance was calculated in a way similar to our previous publications using the following equations. $[2,6,14]$

$$
C_{g r}=\frac{\frac{2}{\Delta E} \int i d t}{m} \quad \text { (1) } \quad ; C_{v o l}=C_{g r} \cdot \rho_{\text {carbon }}
$$

where $\mathrm{C}_{\mathrm{gr}}$ is the gravimetric capacitance, $\mathrm{C}_{\mathrm{vol}}$ is the volumetric capacitance, $\Delta \mathrm{E}$ is the discharge voltage window, $\mathrm{m}$ is the mass of carbon in one electrode, and $\rho_{\text {carbon }}$ is the density of the active slurry.

\section{Results and Discussion}

\subsection{Morphological and structural characterization}

Simultaneous reduction and wrapping of GO sheets on CS was performed hydrothermally at $150{ }^{\circ} \mathrm{C}$ for $6 \mathrm{~h}$ (Fig. 1). Hydrothermal synthesis is considered as facile, 
relatively inexpensive, and does not require the use of any caustic or hazardous materials/processes. [26,27] The use of CS in the rGO@CS hybrid allows us: (1) to maintain the flowability of the active material, (2) provide a conductive support for effective rGO sheet wrapping, (3) avoid rGO sheet aggregation, (4) provide interconnected conductive paths for the rapid charge transport throughout the slurry network, and (5) maintain a high surface area for charge storage.

Figure 2 shows the scanning electron microscope (SEM) micrographs of CS, rGO and as- synthesized hybrid materials. Fig. 2a shows a low magnification image of the phenolic resin derived CS while Fig. 2b shows the higher magnification of the CS representing porous and uniform surface of the CS. Fig. 2c displays morphology of the as-synthesized hybrid (rGO@CS). It is seen from the Fig. 2c that each spherical particle is wrapped with rGO sheets (red arrows) that provide particle to particle contacts to assist rapid charge transport. This interconnected network is important for the charge percolation in the slurries. Thus, improved electrochemical behavior is anticipated for the hybrids. Figure 2d shows a rGO micrograph having crumpled, 2D layered structure with submicrometer voids.

The pore volume, surface area and pore size distributions of the CS and its hybrid with rGO sheets were determined by the nitrogen sorption experiments. Adsorption-desorption isotherms (Fig. 3a) of both materials predominantly demonstrated type IV hysteresis loop at relative pressure between 0 and 1 , showing the characteristics of the presence of the mesopores in the material. The BET specific surface area (SSA) of the CS was found to be $2061 \mathrm{~m}^{2} / \mathrm{g}$, which then reduced to $1325 \mathrm{~m}^{2} / \mathrm{g}$ for rGO@CS hybrid material. This observed decrease in SSA is most likely due to the coverage of the CS by rGO sheets during hydrothermal treatment, which may block some pores. Pore size distributions of both samples (Fig. 3b) reveal that micropores 
are the major contributor to the porosity of the both materials. Pore volume of the CS was found to be $1.1 \mathrm{cc} / \mathrm{mm} / \mathrm{g}$ which was reduced to $0.5 \mathrm{cc} / \mathrm{mm} / \mathrm{g}$ for the as-synthesized hybrid. This decrease may be attributed to the wrapping of the CS with rGO sheets. Although the pore volume of the hybrid sample was reduced, the pore diameter was significantly increased from 0.6 $\mathrm{nm}$ to $0.86 \mathrm{~nm}$ which may provide enhanced transport pathways for ions adsorption. Increase in the pore diameter is attributed to the hydrothermal treatment which may have opened the blocked pores and created some mesopores. The increase in pore size can be seen from the PSD plot, where a prominent peak was observed at $2.5 \mathrm{~nm}$. (Fig. 3b) Nevertheless, as synthesized conductive hybrids of CS and rGO sheets demonstrated high surface area, mixed meso-micro porosity and large pore diameters, required in capacitive slurries for enhanced electrochemical properties.

Hydrothermally induced structural changes were further examined by the Raman spectroscopy (Fig. 3c). Raman spectrum of the GO showed D and G bands at 1330 and $1594 \mathrm{~cm}^{-}$ ${ }^{1}$, respectively. [28] Hydrothermally reduced GO and rGO@CS showed similar D and G band positions except the higher $\mathrm{D}$ band intensity, which confirms the formation of longer $\mathrm{sp}^{2}$ domains. [28] $\mathrm{I}_{\mathrm{D}} / \mathrm{I}_{\mathrm{G}}$ for the $\mathrm{GO}$ was found to be 0.94 , which increased to 1.07 after hydrothermal reduction. This increase can be attributed to the formation of graphitic domains and removal of the oxygen containing functional groups. $\mathrm{I}_{\mathrm{D}} / \mathrm{I}_{\mathrm{G}}$ value of the as-synthesized hybrid was found to be 1.04 (slightly lower than rGO). [28]

FTIR analysis was further performed to probe the changes in the functional groups caused by the GO reduction and during the synthesis of the hybrid materials (Fig. 3d). The characteristic bands for GO were found to be at $~ 3385,2812,1730,1625,1409,1222,1042$ and $980 \mathrm{~cm}^{-1}$, which are assigned to $\mathrm{O}-\mathrm{H}$ vibrations, $\mathrm{C}-\mathrm{H}$ stretching, $\mathrm{C}=\mathrm{O}$ stretching, $\mathrm{C}=\mathrm{C}$ stretching 
of $\mathrm{sp}^{2}$ hybridized backbone, $\mathrm{C}-\mathrm{OH}$ deformation vibrations, $\mathrm{C}-\mathrm{O}$ stretching vibrations of phenolic groups and C-O vibrations from epoxy, ether or peroxide functional groups, respectively. [24] All oxygen associated bands almost disappeared in the FTIR spectrum of the r-GO, as well as in the rGO@CS hybrid, which confirmed the successful reduction of the GO. The appearance of the two new peaks in $\mathrm{r}-\mathrm{GO}$ spectrum at 1548 and $1150 \mathrm{~cm}^{-1}$ can be ascribed to the $\mathrm{sp}^{2}$ hybridized $\mathrm{C}=\mathrm{C}$ skeletal vibrations of $\mathrm{r}-\mathrm{GO}$ sheets. [24] Thermogravimetric analysis (TGA) of GO and rGO

is demonstrated in Fig. 3e to further confirm hydrothermal reduction of GO. GO exhibited a major weight loss between $100-400{ }^{\circ} \mathrm{C}$, which can be ascribed to the loss of water molecules and oxygen-containing functional groups present on the $\mathrm{GO}$ yielding steam, $\mathrm{CO}$ and $\mathrm{CO}_{2}$. In contrast to GO, higher thermal stability of the r-GO confirmed the reduction of GO. [24]

\subsection{Electrochemical characterizations}

The electrochemical behavior of the flowable electrodes composed of CS, pristine rGO, and various compositions of the hybrid (rGO@CS) are investigated using cyclic voltammetry (CV), galvanostatic charge/discharge (GCPL), and electrochemical impedance spectroscopy (EIS) employing a two-electrode set-up.

Cyclic voltammetry measurements were performed on pristine CS flowable electrodes at various scan rates $(2-20 \mathrm{mV} / \mathrm{s})$ within the potential window of 0 to $1 \mathrm{~V}$ in $1 \mathrm{M} \mathrm{H}_{2} \mathrm{SO}_{4}$ (Fig. 4a). Typical rectangular $\mathrm{CVs}$, a characteristic of the capacitive charge storage (double layer formation) or electrosorption of ions, were observed at lower scan rates. CVs become resistive above $20 \mathrm{mV} / \mathrm{s}$, which is most likely due to the diffusion limitations (longer transport pathways) of the ions into the pores and poor particle contacts (connectivity). The capacitance of the pristine CS (based on the electrode mass) at 2, 5, 10, $20 \mathrm{mV} / \mathrm{s}$ was found to be 120, 108, 90 and $60 \mathrm{~F} / \mathrm{g}$, respectively. To overcome the conductivity and connectivity issue of the slurry, various 
ratios of GO to $\mathrm{CS}$ were investigated in the as-synthesized hybrids and their electrochemical performance was further analyzed.

Fig. 4b shows the rate-dependent CVs for the optimized GO to CS composition $(1: 2)$ at various scan rates between $2-20 \mathrm{mV} / \mathrm{s}$. CVs of the hybrid flowable electrodes remained almost rectangular without any distortion even at higher scan rates, indicating rapid charge propagation within the hybrid flowable electrode, which leads to a higher charge storage capability. The capacitance of the optimized flowable electrodes (rGO@CS, 1:2) at $2 \mathrm{mV} / \mathrm{s}$ was found to be 200 $\mathrm{F} / \mathrm{g}$, which is $67 \%$ higher than CS electrodes and $150 \%$ higher than $\mathrm{rGO}(80 \mathrm{~F} / \mathrm{g})$ electrodes at same scan rates. This increase in capacitance may stem from the synergistic effect of the two carbon materials having different physical properties and particle morphology, where one provides a higher surface area for ion adsorption and better rheological performance, and other offers a higher conductivity and facilitates electrical contacts between the CS to enhance the charge percolation throughout the suspension network. Another possible reason for the improved electrochemical performance can be the highly conductive interconnected spherical architecture of the as-synthesized hybrids enables better (and more) utilization of the active material [23].

In order to better understand the significance of the conductivity and connectivity between the particles during the charging/discharging, we compared the CVs of CS and rGO@CS flowable electrodes at $10 \mathrm{mV} / \mathrm{s}$ within the potential range from 0 to $1 \mathrm{~V}$. (Fig. $4 \mathbf{c}$ ). CV of rGO@CS showed almost double the current compared to the CS electrode, suggesting that conductive rGO wrapping on CS accelerates the electronic transport in the flowable electrodes; thus providing effective utilization of both $\mathrm{CS}$ and rGO sheets. In contrast to hybrid flowable electrodes, CS electrodes not only showed lower current values, but also demonstrated distorted $\mathrm{CV}$ which we attribute to the poor conductivity and lower connectivity between the large carbon 
spheres. Nevertheless, addition of the optimal amount of graphene improves the conductivity, enhances the particle contacts and reduces the diffusion distance leading to rapid percolation and enhanced electrochemical performance.

Fig. 4d compares the rate performance of the flowable electrodes composed of pristine $\mathrm{CS}$, pristine $\mathrm{rGO}$ and all the hybrid compositions at various scan rates (between $2-200 \mathrm{mV} / \mathrm{s}$ and between 0 to $1 \mathrm{~V}$ ). The highest capacitance was observed at $2 \mathrm{mV} / \mathrm{s}$ for tested flowable electrodes, while the best rate handling among all the flowable electrodes was observed for the 1:2 composition (rGO@CS). The higher capacitance at lower scan rates is due to the fact that electrolyte ions are capable to penetrate deeper into the carbon pores, providing higher area for the charge storage percolation and leading to higher capacitance, but at higher scan rates, large carbon particles are not fully charged and the ions are mainly limited to the outer surface area or subsurface pores in the CS.

When the scan rate was increased from 2 to $200 \mathrm{mV} / \mathrm{s}$, flowable electrodes comprised of CS, rGO@CS (1:1), rGO@CS (1:2) showed a decrease in capacitance by 91, 84 and 68\%, respectively. The best rate handling was found for the rGO@CS (1:2) hybrid electrode. Furthermore, the interconnected conductive wrapping provides easier pathways for the ions, which yielded a higher capacitance and overall superior rate performance. It is worthwhile to point out that the best composition (1:2) showed a better rate performance when compared with various previously reported flowable electrodes. [5,12-14,17] The highest decline in capacitance for rGO based hybrids was recorded when excess amount (1:1) of the GO was added, which is due to the rGO sheets aggregation/stacking and blocking pores of the CS particles, which limits the accessibility of the electrolyte ions to the CS pores. [23] These findings suggest that GO to 
CS balance is important in producing highly efficient flowable electrodes for the EFC or other related technologies [17,29-31]

Impedance spectroscopy further provided insights into the electrical resistance and diffusion limitation. The Nyquist plots (Fig. 4e) show that all flowable electrodes showed a low interfacial charge resistance, which can be attributed to good connectivity between the CS. All samples exhibited nearly vertical lines in the low-frequency range, which is indicative of primarily capacitive behavior. The sloped region of the Nyquist plot (Fig. 4e, inset), best known as Warburg region, provides evidence about diffusion limitations at low frequencies. The suspension without rGO (bare CS) showed the highest Warburg resistance length, whereas rGO@CS (1:2) showed limited diffusion limitations. The high frequency intercept on the real axis region in the Nyquist is the equivalent series resistance (ESR) or the sum of the interfacial resistance at the active material/current collector and ionic resistance of the electrolyte. All flowable electrodes showed <1 $\Omega$ ESR values. [14] Ragone plot (Fig. 4f) of tested electrode showed that rGO:CS (1:2) composition yielded the energy density of up to $7 \mathrm{Wh} / \mathrm{kg}$, which is $150 \%$ higher than the pristine graphene flowable electrode.

The optimized flow-electrode rGO:CS (1:2) was then studied by chronoamperometry in an intermittent flow-mode (Fig. 5). The capacitance of a $15 \mathrm{wt} \%$ flow electrode was calculated from the discharge curve and found to be $45 \mathrm{~F} \mathrm{~g}^{-1}$, which corresponds to $9.1 \mathrm{~F} \mathrm{~mL}^{-1}$. A lower concentration was used for improved flowability. The obtained capacitance was higher than previously reported various flowable electrodes based on carbon materials (Table 1). [2,13] This clearly indicates that wrapping of CS with rGO sheets is electrochemically beneficial in both static and flow mode regimes. 


\section{Conclusions}

In conclusion, we have shown that enhanced conductivity and connectivity of flowable electrodes leads to improve the electrochemical performance. Wrapping of conductive rGO sheets on CS yielded a higher gravimetric capacitance and rate performance, outperforming all previously reported carbon based flowable electrodes. Various compositions of the CS and GO hybrids (rGO@CS) were produced and tested in static configuration, with the 1:2 (GO: CS) ratio yielding the highest gravimetric capacitance $(200 \mathrm{~F} / \mathrm{g})$, improved rate handling, and the lowest ESR value. The better performance of graphene based suspension electrode is explained by the improved conductivity (rapid percolation in the suspensions), lower rGO sheet aggregation, and interconnected 3-D network which aided to shorten the electron transport paths. The higher amount of rGO (1:1, GO:CS) led to a higher viscosity (poor flowability), higher capacitance retention, and lower gravimetric capacitance. This demonstrated that a balanced rGO-CS ratio is critical for the optimum performance of the flowable electrodes. When tested under intermittent flow configuration, the optimized flowable electrode yields $45 \mathrm{~F} / \mathrm{g}$, outperforming previously reported carbon based electrodes.

\section{Acknowledgement}

Materials development and electrochemical studies were supported as part of the Fluid Interface Reactions, Structures and Transport (FIRST) Center, an Energy Frontier Research Center funded by the U.S. Department of Energy, Office of Science, Basic Energy Sciences under Award no. ERKCC61. ECK acknowledges the support from US National Science Foundation (Grant\# 1351161). KBH was supported by the NSF Graduate Research Fellowship (Grant\# 1002809). Santosh K Yadav is thanked for the TGA analysis. Babak Ansori is 
acknowledged for SEM analysis. The SEM imaging was completed at Drexel's Centralized Research Facilities (CRF).

\section{References}

[1] Huskinson B, Marshak MP, Suh C, Er S, Gerhardt MR, Galvin CJ, et al. A metal-free organic-inorganic aqueous flow battery. Nature 2014;505:195-8.

[2] Presser V, Dennison CR, Campos J, Knehr KW, Kumbur EC, Gogotsi Y. The Electrochemical Flow Capacitor: A New Concept for Rapid Energy Storage and Recovery. Adv Energy Mater 2012;2:895-902.

[3] Simon P, Gogotsi Y. Charge storage mechanism in nanoporous carbons and its consequence for electrical double layer capacitors. Philos Trans A Math Phys Eng Sci 2010;368:3457-67.

[4] Duduta M, Ho B, Wood VC, Limthongkul P, Brunini VE, Carter WC, et al. Semi-Solid Lithium Rechargeable Flow Battery. Adv Energy Mater 2011;1:511-6.

[5] Hatzell KB, Fan L, Beidaghi M, Boota M, Pomerantseva E, Kumbur EC, et al. Composite Manganese Oxide Percolating Networks As a Suspension Electrode for an Asymmetric Flow Capacitor. ACS Appl Mater Interfaces 2014;6:8886-93.

[6] Dennison CR, Beidaghi M, Hatzell KB, Campos JW, Gogotsi Y, Kumbur EC. Effects of flow cell design on charge percolation and storage in the carbon slurry electrodes of electrochemical flow capacitors. J Power Sources 2014;247:489-96.

[7] Wang W, Luo Q, Li B, Wei X, Li L, Yang Z. Recent Progress in Redox Flow Battery Research and Development. Adv Funct Mater 2013;23:970-86.

[8] Brushett FR, Vaughey JT, Jansen AN. An All-Organic Non-aqueous Lithium-Ion Redox Flow Battery. Adv Energy Mater 2012;2:1390-6.

[9] Huang Q, Li H, Grätzel M, Wang Q. Reversible chemical delithiation/lithiation of LiFePO4: towards a redox flow lithium-ion battery. Phys Chem Chem Phys 2013;15:1793-7. 
[10] Sasi S, Murali A, Nair S V., Nair a. S, Subramanian KR V. The effect of graphene on the performance of an electrochemical flow capacitor. J Mater Chem A 2015;3:2717-25.

[11] Porada S, Lee J, Weingarth D, Presser V. Continuous operation of an electrochemical flow capacitor. Electrochem Commun 2014;48:178-81.

[12] Campos JW, Beidaghi M, Hatzell KB, Dennison CR, Musci B, Presser V, et al. Investigation of carbon materials for use as a flowable electrode in electrochemical flow capacitors. Electrochim Acta 2013;98:123-30.

[13] Hatzell KB, Beidaghi M, Campos JW, Dennison CR, Kumbur EC, Gogotsi Y. A high performance pseudocapacitive suspension electrode for the electrochemical flow capacitor. Electrochim Acta 2013;111:888-97.

[14] Boota M, Hatzell KB, Beidaghi M, Dennison CR, Kumbur EC, Gogotsi Y. Activated Carbon Spheres as a Flowable Electrode in Electrochemical Flow Capacitors. J Electrochem Soc 2014;161:A1078-83.

[15] Zhang C, Hatzell KB, Boota M, Dyatkin B, Beidaghi M, Long D, et al. Highly Porous Carbon Spheres for Electrochemical Capacitors and Capacitive Flowable Suspension Electrodes. Carbon 2014;77:155-64.

[16] Hatzell KB, Boota M, Kumbur EC, Gogotsi Y. Flowable Conducting Particle Networks in Redox-Active Electrolytes for Grid Energy Storage. J Electrochem Soc 2015;162:A500712 .

[17] Hatzell KB, Hatzell MC, Cook KM, Boota M, Housel GM, McBride A, et al. Effect of Oxidation of Carbon Material on Suspension Electrodes for Flow Electrode Capacitive Deionization. Environ Sci Technol 2015:150211062348002.

[18] Boota M, Hatzell KB, Kumbur EC, Gogotsi Y. Towards high energy density pseudocapacitive flowable electrodes via incorporation of hydroquinone. ChemSusChem 2015;8:835-43.

[19] Lei Z, Christov N, Zhao XS. Intercalation of mesoporous carbon spheres between reduced graphene oxide sheets for preparing high-rate supercapacitor electrodes. Energy Environ Sci 2011;4:1866.

[20] Peng HJ, Huang JQ, Zhao MQ, Zhang Q, Cheng XB, Liu XY, et al. Nanoarchitectured graphene/CNT@porous carbon with extraordinary electrical conductivity and interconnected micro/mesopores for lithium-sulfur batteries. Adv Funct Mater 2014;24:2772-81.

[21] Yadav SK, Jung YC, Kim JH, Ko Y Il, Ryu HJ, Yadav MK, et al. Mechanically robust, electrically conductive biocomposite films using antimicrobial chitosan-functionalized graphenes. Part Part Syst Charact 2013;30:721-7. 
[22] Yadav SK, Yoo HJ, Cho JW. Click coupled graphene for fabrication of high-performance polymer nanocomposites. J Polym Sci Part B Polym Phys 2013;51:39-47.

[23] Stoller MD, Park S, Zhu Y, An J, Ruoff RS. Graphene-based ultracapacitors. Nano Lett 2008;8:3498-502.

[24] Lindfors T, Boeva Z a., Latonen R-M. Electrochemical synthesis of poly(3,4ethylenedioxythiophene) in aqueous dispersion of high porosity reduced graphene oxide. RSC Adv 2014;4:25279.

[25] Smith KC, Chiang Y-M, Craig Carter W. Maximizing Energetic Efficiency in Flow Batteries Utilizing Non-Newtonian Fluids. J Electrochem Soc 2014;161:A486-96.

[26] Gogotsi YG, Nickel KG, Bahloul-Hourlier D, Merle-Mejean T, Khomenko GE, Skjerlie KP. Structure of carbon produced by hydrothermal treatment of $\beta$-SiC powder. J Mater Chem 1996;6:595.

[27] Basavalingu B, Calderon Moreno JM, Byrappa K, Gogotsi YG, Yoshimura M. Decomposition of silicon carbide in the presence of organic compounds under hydrothermal conditions. Carbon 2001;39:1763-6.

[28] Chen Y, Zhang X, Zhang D, Yu P, Ma Y. High performance supercapacitors based on reduced graphene oxide in aqueous and ionic liquid electrolytes. Carbon 2011;49:573-80.

[29] Porada S, Weinstein L, Dash R, van der Wal a, Bryjak M, Gogotsi Y, et al. Water desalination using capacitive deionization with microporous carbon electrodes. ACS Appl Mater Interfaces 2012;4:1194-9.

[30] Hatzell KB, Iwama E, Ferris A, Daffos B, Urita K, Tzedakis T, et al. Capacitive deionization concept based on suspension electrodes without ion exchange membranes. Electrochem Commun 2014;43:18-21.

[31] Hatzell MC, Hatzell KB, Logan BE. Using Flow Electrodes in Multiple Reactors in Series for Continuous Energy Generation from Capacitive Mixing. Environ Sci Technol Lett 2014;1:474-478. 


\section{FIGURES}



Carbon Spheres

Figure 1: Schematic presentation of the

synthesis of highly conductive hybrid of rGO sheets wrapped on carbon spheres (rGO@CS) and suspension electrode preparation. 

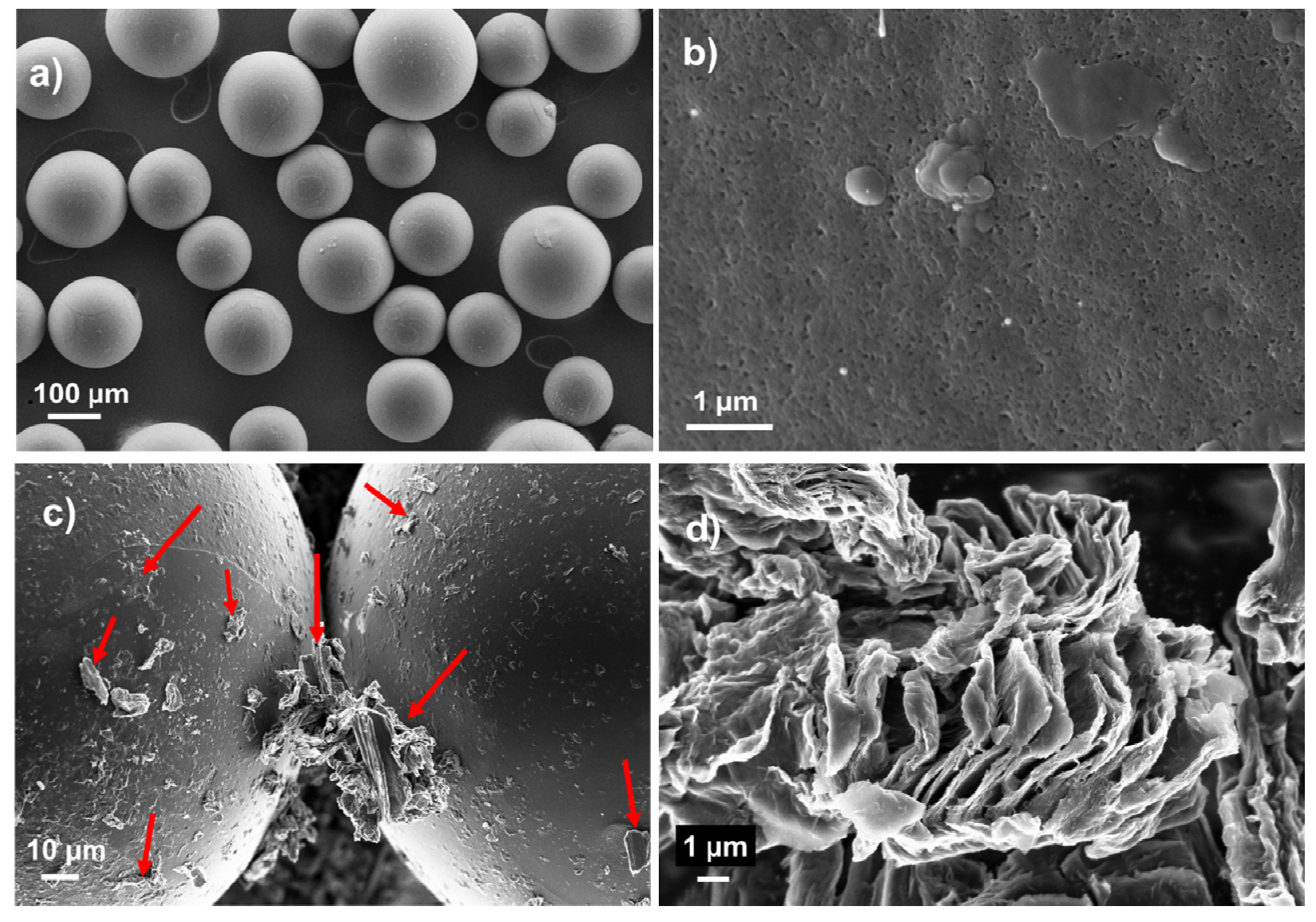

Figure 2: (a) Low magnification SEM image of carbon spheres (b) High magnification single carbon sphere surface (c) SEM image of spheres contact and wrapping with rGO sheets. (d) Crumpled open structure of rGO with open voids. 

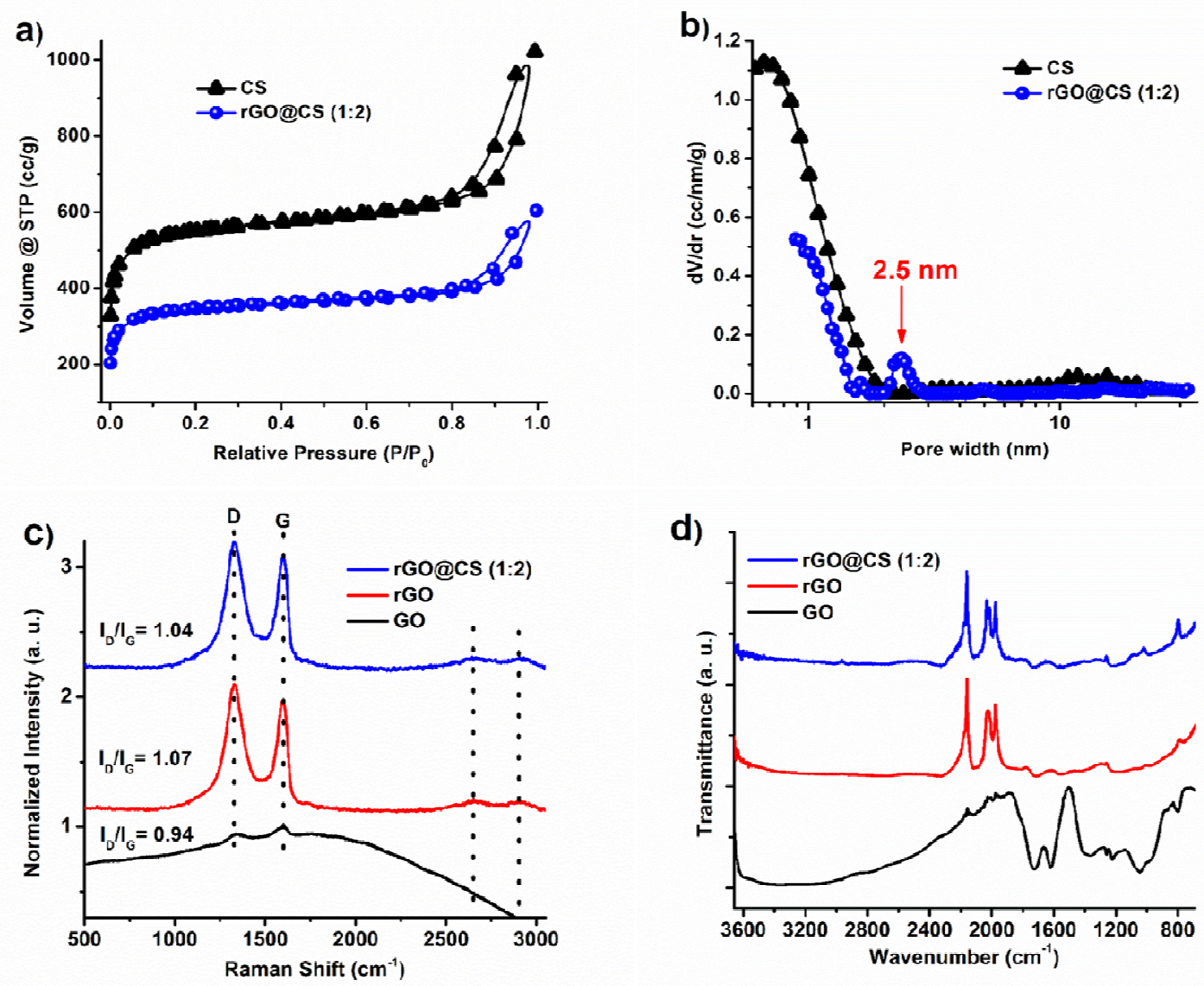

Figure

Nitrogen gas sorption rGO@CS (1:2),

(b)

size distributions,

(c)

rGO and hybrid

Corresponding Fourier e)



3: (a)

isotherms for CS and Corresponding pore Raman spectra of GO, rGO@CS (1:2), Transform Infrared spectra, (E) TGA curves of GO and rGO in Argon at $2.5^{\circ} \mathrm{C} / \mathrm{min}$. 



Figure 4: (a) Cyclic voltammograms of CS flowable electrodes, (b) CVs of the rGO@CS optimized composition (c) CV comparison of the CS and hybrid (rGO@CS, 1:2) flowable electrode at $10 \mathrm{mV} / \mathrm{s}$ rate, (d) Rate performance of the various flowable electrodes, (f) Nyquist plots for the tested flowable electrodes, and (f) corresponding Ragone plot. 


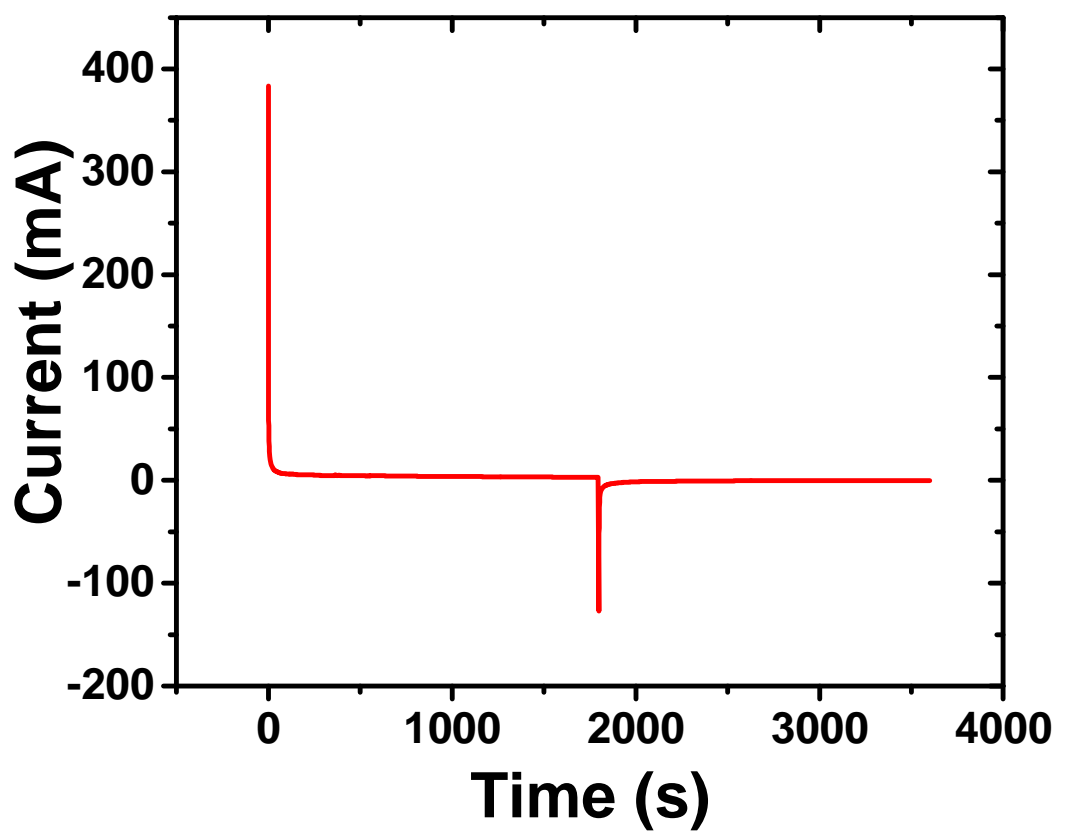

Figure 5: Chronoamperometric plot of the optimal composition (rGO@CS, 1:2) flowable electrode (15 wt \% solid fraction) in the intermittent flow mode. 